\title{
Empirical Validation of the RCDC and RCDE Semantic Complexity Metrics for Object-oriented Software
}

\author{
Glenn W. Cox ${ }^{1}$, Sampson E. Gholston ${ }^{2}$, Dawn R. Utley ${ }^{2}$, Letha H. Etzkorn ${ }^{1}$, \\ Cara Stein Gall ${ }^{3}$, Phillip A. Farrington ${ }^{2}$ and Julie L. Fortune ${ }^{2}$ \\ ${ }^{1}$ Department of Computer Science, University of Alabama in Huntsville, USA \\ ${ }^{2}$ Industrial and Systems Engineering Management Department, University of Alabama in Huntsville, USA \\ ${ }^{3}$ Department of Computer Science, Edinboro University of Pennsylvania, USA
}

The Relative Class Domain Complexity (RCDC) and Relative Class Definition Entropy (RCDE) semantic metrics have been proposed for use as complexity metrics for object-oriented software. These semantic metrics are calculated on a knowledge-based representation of software, following a knowledge-based program understanding examination of the software. The metrics have great potential because they can be applied during the software design phase whereas most complexity metrics cannot be applied until after development is complete. In this paper, we present the results of a study to empirically validate the RCDC and RCDE metrics. We show that the metrics compare favorably with the findings of human experts and also that they correlate well with the results of conventional complexity metrics.

Keywords: object-oriented, metrics, semantic metrics, complexity, entropy

\section{Introduction}

Object-oriented software developers widely consider the complexity of code to be one of the top factors determining software cost, reliability, and main-tainability. Because of this, code complexity is probably the most commonly estimated software quality, and complexity metrics are included in nearly every metrics suite used for object-oriented design (OOD).

The majority of the complexity metrics used in OOD are based on the concept that the "complexity of an object can be defined to be the cardinality of its set of properties" [1]. Despite this common foundation, because different metrics designers have focused on different types of properties, there is a wide variety of complexity metrics. For example, one complexity metric the Weighted Methods per Class (WMC) metric [1] - simply assigns a number to represent the complexity of each method in a class, then sums the numbers. Another metric, McCabe's Cyclomatic Complexity (CC) metric [2] actually attempts to count the number of independent paths through a segment of code.

Most complexity metrics measure characteristics of the code's syntax. These syntacticallybased metrics have certain problems that limit their usefulness. First, they can be biased by insignificant differences in coding style. For example, it has been shown [3] that small coding choices, such as placing multiple functions in a single line of code rather than multiple lines, can yield different syntactical metrics results, although there is no difference in the complexity of the function performed.

A second problem with syntactic metrics is that nearly all of them are calculated from the characteristics of implemented code, meaning that they cannot be applied until fairly late in the development process. A subclass of syntactic metrics, called Design Metrics, is an attempt to address this shortcoming. Design metrics, as implied by the name, measure characteristics derived from a program's design allowing them to be applied before the start of coding. Design metrics are valuable compared to code metrics because the earlier in the development cycle problems can be found, the less costly these problems are to fix [4]. 
Common design metrics measure characteristics of the class-level structure of the program. Metrics of this type include the number of classes in the system, the number of functions in the system, etc. Obviously, these metrics are limited in the amount of information they can give about the complexities of individual classes.

A more detailed design metric is Depth of Inheritance Tree (DIT), which examines the inheritance relationship between classes in a system. Although this metric does examine the impact of inheritance on a particular class's complexity, it is perhaps more useful in providing a systemwide view of class relationships than it is in giving an examination of per class complexity. Another well-known design metric (a variant of the WMC metric) counts the member functions of a class.

While these and other syntactically-based design metrics are capable complexity metrics, they suffer from some of the problems common to most syntactic metrics. For example, they are sensitive to stylistic design differences that human evaluators would consider to be insignificant. Also, most of the conventional design metrics are intended for use at a structural level and give little specific information about particular portions of the system that might have poor designs.

Etzkorn and others addressed these problems by developing the field of Semantic metrics. Instead of measuring the syntactic characteristics of a module, semantic metrics use knowledgebased program understanding and natural language processing techniques to analyze the functions performed by a program, independent of the way the functions are coded. This earlier work included development of two suites of semantic complexity metrics for $\mathrm{OO}$ designs. The first was called Relative Class Domain Complexity (RCDC) metrics [5] and the second, Relative Class Definition Entropy (RCDE) metrics [3].

This paper presents the results of an effort to validate the RCDC and RCDE metrics. The validation was performed by comparing the results of the semantic metrics with: (1) complexity estimates produced by software development experts and (2) and results from two conventional syntactic metrics, WMC and the Sum of the Static Complexities of Local Methods (SSCLM). (SSCLM is sometimes referred to as "WMC-McCabe". In this paper, we use the name "Sum of the Static Complexities of Local Method" to better clarify the basis on which the metric is calculated.)

The software on which the comparison was based consisted of 40 classes extracted from two widely-used object-oriented Graphical User Interface (GUI) packages, GINA [6] and wxWindows [7] and a widely used matrix math library, Newmat [8]. The semantic metrics were automatically generated by the SemMet metrics package, a Semantic Metrics generation tool developed by the University of Alabama in Huntsville for the National Aeronautics and Space Administration (NASA). The three sets of results were then correlated to provide a statistical comparison of the different complexity estimation approaches.

The following sections present descriptions of the metrics analyzed in this study, the analytical results, and conclusions and future research directions.

\section{Background}

This section overviews the program understanding approach and tools used in the study, and defines the metrics that were validated

\subsection{Program Understanding Tool}

Calculation of semantic metrics requires some degree of program understanding in order to determine the semantics underlying the specifics of the program. The SemMet tool is based on an automatic program understanding tool called the PATRicia system $[9,10]$. The PATRicia system understands the functionality of a program in order to analyze components in Object-Oriented software. It uses a heuristic approach, deriving information from the linguistic aspects of comments and identifiers, and from other non-linguistic aspects of object-oriented software, such as a class hierarchy. A complete description of the operation of the PATRicia system is available in [11].

The program understanding and information extraction module of the PATRicia system is called CHRiS (Conceptual Hierarchy for Reuse including Semantics). A data flow diagram illustrating the operation of CHRiS is shown in 
Figure 1 [11]. Among other functions, CHRiS is the module that understands program comments. When analyzing comments, CHRiS parses a sentence using a simple natural language parser, then uses an inference engine for semantic processing. For identifiers, CHRiS uses empirical information on common formats for variable and function identifiers to syntactically tag subkeywords.

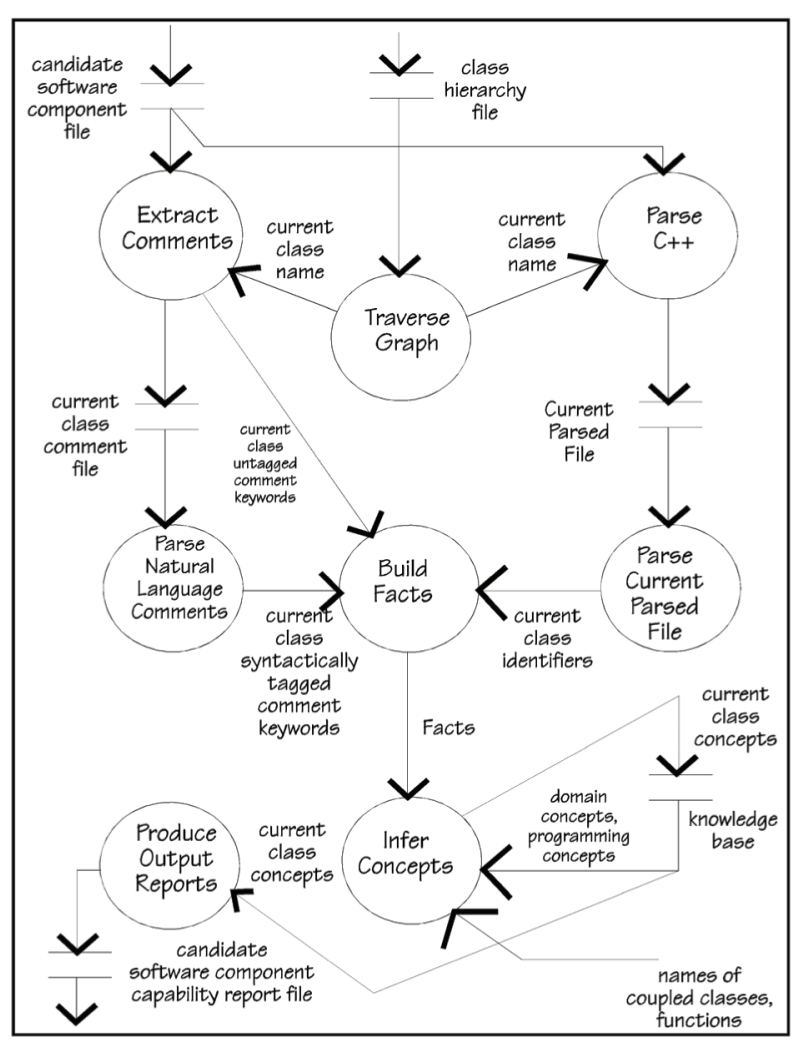

Figure 1. Operation of the CHRiS module of the PATRicia system [11].

CHRiS uses a weighted, hierarchical semantic network (a structure that represents knowledge as a pattern of interconnected nodes and arcs) in which higher-level concepts are inferred from lower level concepts. Inference occurs through a form of spreading activation, where active nodes spread to, or infer, surrounding nodes. Natural language tokens from comments and identifiers are applied to an interface layer of the semantic net, which consists of syntactically tagged keywords, and from this layer inference proceeds to the rest of the semantic net.

The semantic network is object-oriented. It is implemented as objects and messages in the expert system shell of CLIPS version 6.0. In CHRiS, a concept is implemented as one or more CLIPS objects. When a concept is asserted, weighted messages are sent from that concept to surrounding concepts, and a comparison of the weights provides an inference mechanism. The CHRiS module of the PATRicia system has been satisfactorily validated [9]

CHRiS produces a report that identifies concepts and keywords that have been found in each examined class. The report shows the number of times each identified keyword was found. It also shows the number of times each concept (from interior conceptual graphs) was inferred from the inference engine of the semantic network.

\subsection{Representing Semantic Information}

In SemMet, semantic information is represented using Conceptual Graphs, a widely-used knowledge representation technique [12]. A conceptual graph is a finite, connected, bipartite graph that contains two different types of nodes, concepts and conceptual relations. A concept is an internal representation of an entity. Conceptual relations specify the role that a concept plays with respect to another concept. Arcs in a conceptual graph form links between concepts and conceptual relations.

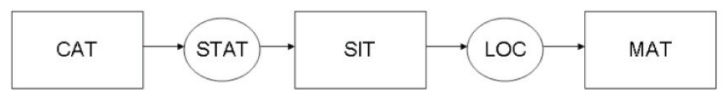

Figure 2. Conceptual graph example [12].

A frequently used example of a conceptual graph is shown in Figure 2. This conceptual graph states that a Cat is Sitting on a Mat. Specifically, the conceptual graph states that the concept"cat" has the state "sit" at the location "mat".

Detailed information about semantic graphs can be found in reference [12].

\subsection{Mathematical Definition of the RCDC Metrics}

The RCDC and RCDE metrics suites were originally defined by Etzkorn et. al. in references [3] and [5]. Stein [13] developed a formal mathematical definition of the suites. For 
the reader's convenience, the mathematical definition is summarized in this and the following section. A more complete definition can be found in references [13] and [14].

The following mathematical notation is used to define the RCDC metrics. Let $\left\{C_{1}, C_{2}, \ldots, C_{m}\right\}$ denote the set of $m$ classes in a system. For each class $C_{a}, O_{a}$ denotes the set of concepts in the knowledge base associated with $C_{a}$ and $K_{a}$ is the set of keywords in the knowledge base associated with $C_{a}$. Let $O$ be the set of all concepts in the knowledge base. Let $I_{a}=K_{a} \cup O_{a}$; this is called the set of ideas associated with class $C_{a}$.

Let $\rightarrow$ be a relation between concepts $p$ and $q$ in set $O$ such that $p \rightarrow q$ if and only if there exists a conceptual relation from $p$ to $q$.

The RCDC metrics are based on the Class Domain Complexity (CDC) semantic metric defined by Etzkorn and Delugach [5].

The CDC metrics make use of semantic relationships between the concepts to estimate the tightness of the internal coupling within a class. Specifically, CDC is based on the count of the number of conceptual relations that link each concept with other concepts in the class. It is defined by:

CDC counts the concepts and their associated conceptual relations, and multiplies the counts by a weighting factor for each concept. The suggested scale for concept weighting factors ranges from 1.0 for complex concepts to 0.25 for simple concepts [5].

Three versions of the CDC metric have been developed, each of which uses a slightly different method to calculate the metric. The versions are:

- $C D C 0=\sum_{i \in O_{a}}\left(1 * w_{i}\right)$,

where $w_{i}$ is the weighting factor for concept $i$. This metric simply sums the complexity weightings for the concepts in the class [13]

- $C D C 1=\sum_{i \in O_{a}}\left(\left(1+\left|\left\{x \mid i \rightarrow x \wedge x \in O_{a}\right\}\right|\right) * w_{i}\right)$

This metric determines, for each concept in the class, the number of other concepts that have a conceptual relation with it, and takes the weighted sum across all of the concepts in the class $[5,13]$.

- $C D C 2=\sum_{i \in I_{a}}\left(\left(1+\left|\left\{x \mid i \rightarrow x \wedge x \in I_{a}\right\}\right|\right) * w_{i}\right)$,
This metric extends the CDC1 definition to include keywords identified from the class [14].

The CDC metrics can be viewed as giving an absolute estimate of complexity. In some cases, there is value in considering the complexity of a class with respect to the complexity of the other classes in the same system. This is the goal of the Relative Class Domain Complexity (RCDC) metrics suite [5].

There are RCDC metrics that correspond to each of the CDC metrics. In general, the RCDC metrics are generated by calculating the $\mathrm{CDC}$ metric, then normalizing by the maximum $\mathrm{CDC}$ for all of the classes in the system. That is:

$R C D C x$ for class $i=C D C x$ for class $i /$ maximum $C D C x$ for any class in the system

where $x$ is 0,1 , or 2 . If all CDCx's in the system are $0, R C D C x$ is defined to be 0 .

It should be noted that the RCDC definition is specific for a knowledge base with a conceptual graph structure.

\subsection{RCDE Metrics}

The RCDE metrics are based on the Semantic Class Definition Entropy (SCDE) metric defined by Etzkorn, Gholston, and Hughes [3]. SCDE is a complexity metric that uses information theoretic and program understanding approaches to assess the domain information content of a class. It reflects the complexity of the task that the class performs in the domain (that is, the complexity of the task, if it was performed by a human).

SCDE is defined by:

$$
S C D E=-\sum_{i=1}^{n}\left[\left(f_{i} / N\right) \log _{2}\left(f_{i} / N\right)\right]
$$

where $n$ is the number of unique domain-related concepts or keywords, $N$ is the total number of (non-unique) domain related concepts or keywords, and $f_{i}, 1 \leq i \leq n$ is the frequency of occurrence of the ith domain-related concept or keyword.

Two other versions of the SCDE metric, SCDEa and SCDEb, are calculated identically to SCDE except that SCDEa considers only keywords in the calculation and SCDEb considers only concepts. 
The Relative Class Definition Entropy (RCDE) metric expresses the SCDE of a class relative to the other classes in the same system. RCDE is defined by:

RCDE for class $i=S C D E$ of class $i /$ maximum SCDE for any class in the system

RCDEa and RCDEb are calculated in the same way from SCDEa and SCDEb:

$R C D E a=S C D E a$ of the class $/$ max SCDEa for any class in the system

$R C D E b=S C D E b$ of the class $/$ max SCDEb for any class in the system

\subsection{Summary of Previous Theoretical Analyses of RCDC and CDC}

A theoretical analysis for CDC was presented in reference [5]. Other theoretical analyses for CDC0 and CDC2, as well as for RCDC, were documented in reference [14]. These works showed that all versions of CDC meet the criteria for valid metrics defined by Kitchenham et al. [15] as well as the following properties of Briand et al. [16]: Non-negativity, symmetry, and monotonicity (All versions of CDC fail the null value property since they have a nonzero value for any class associated with even a single idea in the domain, even if there are no relationships within the class.) Although the RCDC metrics are measures of relative complexity, and not of complexity, they fulfill the properties of non-negativity and null value.

Stein [13] performed a theoretical analysis of RCDE and SCDE. She showed that all three versions of SCDE meet the criteria of nonnegativity, null value, and symmetry but do not meet either the disjoint module additivity nor monotonicity criteria. The RCDE metrics meet the same criteria as RCDC.

\section{Validation Procedure and Results}

The metrics were validated by comparing their results for a representative set of classes with independently generated complexity ratings developed by human experts. Forty (40) classes were chosen from widely used open-source packages to form a representative mix of scientific processing types. Twenty three (23) of the classes were drawn from the Newmat $\mathrm{C}++$ software package, which is an open-source mathematical application for matrix manipulation [8]. The selected Newmat classes include code for both matrix and scalar processing. The other 17 classes were drawn from two Graphical User Interface (GUI) packages, GINA and wxWindows. These classes include a wide range of processes for GUI processing techniques, graphical user input, and display management routines.

The validation project was structured as three independent experiments. For each experiment, the SemMet tool was used to generate the RCDC and RCDE metrics for a selected subset of the 40 classes. The same classes were provided to a team of software developers for independent rating of the class's complexity. Each team member rated the complexity of each class using a numeric scale and the results were averaged for each class and for each team. The RCDC and RCDE results were then correlated with the team's ratings.

Experiment 1 focused on the 17 classes from the two GUI packages. The human team (referred to as Evaluation Team 1) was made up of seven software engineering experts. Each of the members of the team has a B.S. in computer science or electrical engineering and all but one also have an M.S. degree. Each has from 5-15 years of experience in software design and development and at least 3 years experience with $\mathrm{C}++$ and GUI development. None of the team members were initially familiar with the systems examined.

Experiment 2 concentrated on the 13 wxWindows classes. The human team, Evaluation Team 2, was composed of 15 graduate students drawn from a graduate-level software engineering class. Each of the members of the team had prior experience in object-oriented software, particularly $\mathrm{C}++$, and most had several years of experience in software design and development. By covering many of the same classes as Experiment 1, Experiment 2 helped verify Experiment 1 results as well as adding information about the performance of the metrics for the wxWindows classes.

Experiment 3 focused on the 23 Newmat classes. The human raters, Evaluation Team 3, had the same characteristics as Evaluation Team 2. There was not any overlap in membership between the teams. 
In each experiment, team members rated the complexity of each class on a scale from 0 to 1 , where "not complex" $=1.00$, "fairly complex" $=0.50$, and "very complex" $=0.00$. The numeric ratings were combined to compute an average expert rating for each class. Group inter-rater reliability for Evaluation Team 1 was 0.9038 , for Evaluation Team 2 was 0.4160 , and for Evaluation Team 3 was 0.5208 .

In addition to correlating RCDC and RCDE with the human experts, the two semantic metrics were also correlated with SSCLM. SSCLM is a traditional (syntactic) development-phase complexity metric $[10]$ that is an advanced version of Chidamber and Kemerer's WMC metric [1]. SSCLM is calculated by

$$
S S C L M=\sum_{i=1}^{n} c_{i}
$$

where $n$ is the number of methods in the class and $c_{i}$ is the McCabe's Cyclomatic Complexity [2] of the $\mathrm{i}^{\text {th }}$ method. In a previous study [10], Etzkorn has shown that SSCLM provides a good measure of the complexity of an object-oriented class and is superior, in fact, to the widely-used WMC metric.

A statistical analysis was performed to correlate the metrics with each other and to gain insight into the relevance of common factors considered by the metrics. Comparing metrics to other metrics using a correlation is a common research method. For example, Kabaili et al. [18] correlated cohesion metrics with coupling metrics to validate cohesion metrics as changeability indicators. Mitchell and Power [18] correlated run time coupling metrics with a static coupling metric to examine the usefulness of the run time coupling metrics. Li and Henry [19] examined correlations between various metrics in order to determine their usefulness within a multi-variate regression model.

\subsection{Complexity Metrics Compared to Evaluation Team Ratings}

The analysis was based on the hypotheses:

$H_{0}: p=0$ (Null hypothesis) - There is no significant correlation between the specified complexity metric and the average rating produced by the specified Evaluation Team. $H_{1}: p \neq 0$ (Alternative hypothesis) - There is significant correlation between the speci- fied complexity metric and the average rating produced by the specified Evaluation Team.

Correlation was measured using the standard Pearson's correlation coefficient, which measures the strength of the linear relationship between the two variables. The coefficient ranges between -1 and 1 , where 1 corresponds to perfect correlation between the two variables, -1 corresponds to perfect negative correlation, and 0 indicates there is no linear relationship. The strength of the correlation is determined by the magnitude of the coefficient. Adjective ratings of correlation strength follow the definitions developed by Hopkins [20] and Cohen [22]:
- $<0.1$
"Trivial"
- 0.1 to 0.3
"Minor"
- 0.3 to 0.5
"Moderate"
- 0.5 to 0.7
"Large"
- 0.7 to 0.9
"Very large"
- 0.9 to 1
"Almost perfect"

The quality of the correlation results was assessed by calculating the p-value for each calculation (the p-value corresponds to the probability that the measured correlation could be due to purely random effects). Correlation results with $\mathrm{p}$-values greater than 0.10 were rejected.

Table 1 shows the results of correlating the metrics results with the human-generated complexity ratings. The results for Experiment 1 show that RCDC0 and RCDC1 have moderate correlation with the expert ratings. RCDEa has large correlation in both Experiments 1 and 2, and moderate correlation in Experiment 3.

The results indicate that the RCDEa metric is a statistically valid measure of complexity for the entire test suite.

\begin{tabular}{|l||c|c|c|c|c|c|}
\hline & \multicolumn{2}{|c|}{$\begin{array}{c}\text { Experiment } \\
\mathbf{1}\end{array}$} & \multicolumn{2}{|c|}{$\begin{array}{c}\text { Experiment } \\
\mathbf{2}\end{array}$} & \multicolumn{2}{|c|}{$\begin{array}{c}\text { Experiment } \\
\mathbf{3}\end{array}$} \\
\hline & $\begin{array}{c}\text { Corr. } \\
\text { vs. } \\
\text { Team } \\
\mathbf{1}\end{array}$ & $\begin{array}{c}\text { p- } \\
\text { value }\end{array}$ & $\begin{array}{c}\text { Corr. } \\
\text { vs. } \\
\text { Team } \\
\mathbf{2}\end{array}$ & $\begin{array}{c}\text { p- } \\
\text { value }\end{array}$ & $\begin{array}{c}\text { Corr. } \\
\text { ves. } \\
\text { Team } \\
\mathbf{3}\end{array}$ & $\begin{array}{c}\text { p- } \\
\text { value }\end{array}$ \\
\hline \hline RCDC0 & -0.43 & 0.09 & -0.40 & 0.17 & -0.09 & 0.65 \\
\hline RCDC1 & -0.43 & 0.08 & -0.42 & 0.15 & -0.11 & 0.63 \\
\hline RCDC2 & -0.40 & 0.11 & -0.44 & 0.13 & -0.19 & 0.40 \\
\hline RCDE & -0.38 & 0.13 & -0.36 & 0.23 & -0.18 & 0.40 \\
\hline RCDEa & -0.51 & 0.04 & -0.58 & 0.04 & -0.39 & 0.07 \\
\hline RCDEb & -0.22 & 0.40 & -0.15 & 0.61 & 0.00 & 0.98 \\
\hline
\end{tabular}

Table 1. Correlation of Complexity metrics with Expert ratings. 
It may be noted that there is good consistency between the ratings generated by the two teams with overlapping focus (Evaluation Teams 1 and 2 ); a correlation of the teams' results gives a correlation coefficient of 0.83 (very large). To simplify the presentation, in the following parts of this paper, the results from these two teams are combined.

\subsection{RCDC and RCDE vs. SSCLM}

Following the validation effort, the performance of the RCDC and RCDE semantic metrics was compared with that of the SSCLM syntactic metric. The results are shown in Tables $2 \mathrm{a}$ and $2 b$.

For both the GUI and math packages, the results show a "large"-to-"very large" correlation between the RCDEa and SSLCM metric and "moderate"-to-"large" correlation between SSLCM and both RCDC2 and RCDE. This is a key result, since the RCDC and RCDE metrics can be applied in the design phase while SSCLM is a development-phase metric.

\begin{tabular}{|l||c|c|c|c|}
\hline \multicolumn{1}{|c||}{} & \multicolumn{2}{c|}{$\begin{array}{c}\text { Experiment 1 } \\
\text { 2 }\end{array}$} & \multicolumn{2}{c|}{ Experiment 3 } \\
\hline & $\begin{array}{c}\text { Corr. vs. } \\
\text { SSCLM }\end{array}$ & $\begin{array}{c}\text { p- } \\
\text { value }\end{array}$ & $\begin{array}{c}\text { Corr. vs. } \\
\text { SSCLM }\end{array}$ & $\begin{array}{c}\text { p- } \\
\text { value }\end{array}$ \\
\hline \hline RCDC0 & 0.60 & 0.01 & 0.30 & 0.17 \\
\hline RCDC1 & 0.57 & 0.02 & 0.28 & 0.20 \\
\hline RCDC2 & 0.58 & 0.01 & 0.45 & 0.03 \\
\hline RCDE & 0.54 & 0.03 & 0.48 & 0.02 \\
\hline RCDEa & 0.64 & 0.01 & 0.72 & 0.00 \\
\hline RCDEb & 0.46 & 0.07 & -0.12 & 0.61 \\
\hline
\end{tabular}

Table 2. Correlation of Complexity metrics with SSLCM.

RCDEa - and to a lesser extent, RCDC2 and RCDE - can consequently be understood as early predictors of the complexity of a class. They provide a way to manage class complexity as a part of the design effort, when cost-effective changes can be implemented.

\subsection{Complexity Metrics Compared to Each Other}

In a third analysis, the results of each metric were correlated with the results of every other metric. The objective of this analysis was to identify possible commonalities in the performance of the various metrics.

The results of the study are shown in Tables $3 \mathrm{a}$ and $3 \mathrm{~b}$. The results show almost perfect correlation between the three RCDC metrics (RCDC0, $\mathrm{RCDC} 1, \mathrm{RCDC} 2)$. This is a surprising result since it indicates that the simple metric RCDC0 - a metric that merely sums complexity weighting factors - provides results that are consistent with the complex RCDC1 and RCDC2 metrics, which count concept and/or keyword linkages. If this is found to be borne out by large-scale studies focused on this specific issue, it may be possible to apply quite simple calculation techniques to the sophisticated semantic complexity metrics.

\begin{tabular}{|c|c|c|c|c|c|}
\hline & RCDCO & RCDC1 & RCDC2 & RCDE & RCDEa \\
\hline RCDC1 & 0.995 & & & & \\
\hline RCDC2 & 0.98 & 0.98 & & & \\
\hline RCDE & 0.80 & 0.79 & 0.81 & & \\
\hline RCDEa & 0.83 & 0.82 & 0.85 & 0.85 & \\
\hline RCDEb & 0.40 & 0.41 & 0.42 & 0.63 & 0.31 \\
\hline
\end{tabular}

Table 3a. Correlation of Complexity metrics with each other (Experiment 1 and 2).

\begin{tabular}{|c|c|c|c|c|c|}
\hline & RCDCO & RCDC1 & RCDC2 & RCDE & RCDEa \\
\hline RCDC1 & 0.995 & & & & \\
\hline RCDC2 & 0.97 & 0.97 & & & \\
\hline RCDE & 0.96 & 0.96 & 0.99 & & \\
\hline RCDEa & 0.42 & 0.43 & 0.62 & 0.61 & \\
\hline RCDEb & 0.79 & 0.77 & 0.65 & 0.64 & -0.10 \\
\hline
\end{tabular}

Table $3 b$. Correlation of Complexity metrics with each other (Experiment 3).

\subsection{Principal Component Analysis}

A Principal Component Analysis (PCA) was performed in order to determine Principal Components that characterize the variance in the empirical data. When metrics are strongly corre- 
lated, it is likely that they measure the same class properties. PCA is a standard technique that has the objective of grouping the data set into a smaller set of orthogonal groups. The groups are formed so that the members of a group have similar variance characteristics and, presumably, similar underlying measurement characteristics $[21,22]$. When using PCA, the larger the sample size the better. It has been recommended that an acceptable sample size has ten times the number of observations as there are variables being analyzed [17]. The data set utilized in this research exceeded the suggested ten times number and therefore the results of the principle component analysis are regarded as valid.

Table 4a shows the PCA results for Experiments 1 and 2. Principal Component 1 (PC1), the members of which are indicated by bold numbers in the PC1 column, includes all of the RCDC and RCDE metrics. This is consistent with the results of the Experiment 3 PCA (Table $4 \mathrm{~b})$, for which those metrics, with the exception of RCDEa, were also grouped.

PCA is a tool that suggests similar underlying measurement characteristics, but does not by itself yield conclusive results. For definitive results, further statistical analysis to validate the groupings is required. However the results of Principal Components analysis do provide useful indicators of commonalities between metrics and help define further analysis.

\begin{tabular}{|l||l|l|}
\hline & PC1 & PC2 \\
\hline \hline Eigenvalue & 7.974 & 2.010 \\
\hline Percent & $66.45 \%$ & $16.75 \%$ \\
\hline Cumulative Percent & $66.45 \%$ & $83.19 \%$ \\
\hline RCDC0 & $\mathbf{0 . 9 6}$ & -0.12 \\
\hline RCDC1 & $\mathbf{0 . 9 6}$ & -0.12 \\
\hline RCDC2 & $\mathbf{0 . 9 7}$ & -0.11 \\
\hline RCDE & $\mathbf{0 . 9 7}$ & -0.14 \\
\hline RCDEa & $\mathbf{0 . 8 5}$ & -0.41 \\
\hline RCDEb & $\mathbf{0 . 8 8}$ & 0.06 \\
\hline SSCLM & $\mathbf{0 . 7 3}$ & -0.03 \\
\hline Complexity (Team 1) & -0.21 & $\mathbf{0 . 9 1}$ \\
\hline Complexity (Team 2) & -0.29 & $\mathbf{0 . 9 1}$ \\
\hline WMC & $\mathbf{0 . 9 5}$ & -0.04 \\
\hline
\end{tabular}

Table 4a. Results of Principal Component Analysis (Experiments $1 \& 2$ ).

\begin{tabular}{|l||l|l|}
\hline & PC1 & PC2 \\
\hline \hline Eigenvalue & 5.3167 & 2.2788 \\
\hline Percent & $53.17 \%$ & $22.79 \%$ \\
\hline Cumulative Percent & $53.17 \%$ & $75.96 \%$ \\
\hline RCDC0 & $\mathbf{0 . 9 9}$ & -0.13 \\
\hline RCDC1 & $\mathbf{0 . 9 9}$ & -0.13 \\
\hline RCDC2 & $\mathbf{0 . 9 3}$ & -0.36 \\
\hline RCDE & $\mathbf{0 . 9 2}$ & -0.38 \\
\hline RCDEa & 0.30 & -0.41 \\
\hline RCDEb & $\mathbf{0 . 8 6}$ & 0.35 \\
\hline Complexity (Team 3) & 0.02 & $\mathbf{0 . 5 3}$ \\
\hline WMC & 0.15 & -0.04 \\
\hline SSCLM & 0.18 & -0.81 \\
\hline
\end{tabular}

Table 4b. Results of Principal Component Analysis (Experiment 3).

In this study, the Principal Components Analysis results lend support to the view that the RCDC and RCDE metrics have commonalities in their underlying measurement characteristics. This result, if borne out in detailed studies, would be significant because of the large variance in the complexity of the various metrics; finding that the simpler metrics measure the same or similar features as the more complex ones could greatly simplify the complexity estimation process.

\section{Conclusions and Further Work}

The validation results show that three of the metrics produce statistically valid complexity ratings, correlating with expert ratings at the "large" and "moderate" levels. The remaining metrics correlate to a lesser degree, but none were found to correlate poorly. Data are not sufficient to determine with certainty that all of the metrics would correlate well with the expert data. However, since there is a very high degree of correlation among the entire set of metrics, it is to be expected that all of the metrics correlate well. In future work, the authors will expand the number of classes for which human ratings are available, allowing a more comprehensive validation.

Each of the metrics were found to correlate well with the traditional SSCLM metric. This is an 
important result, since the RCDC and RCDE metrics can be applied earlier in the software development process than SSCLM. The results indicate that the semantic metrics can be used as predictors of class complexity from design information alone, facilitating early correction of poor class design practices.

Another important finding is that the three RCDC-based metrics (RCDC0, RCDC1, and RCDC2) are almost perfectly correlated, despite considerable conceptual differences in the way they are calculated. In future work, the authors will conduct detailed investigations of this apparent similarity between the three metrics. If consistent results are found, it may be practical to compact the set of RCDC metrics.

Unlike the RCDC metrics, it was found that the entropy-based RCDE metrics yield considerably different results among themselves. The indication is that concepts may be slightly underweighted in RCDE relative to keywords and that gaining a comprehensive understanding of the characteristics requires generating $\mathrm{RCDEb}$ in addition to one of the other RCDE metrics.

\section{Acknowledgement}

The research in this paper was partially supported by NASA grants NAG5-12725 and NCC8-200.

\section{References}

[1] S. Chidamber, C. Kemerer, Towards a metrics suite for object-oriented design.Proceedings of the Conference on Object-Oriented Programming: Systems, Languages and Applications, (1991), pp. 197-211.

[2] T. MCCABE, C. Butler, Design complexity measurement and testing. Communications of the Association for Computing Machinery (ACM), 32 (1989), 12, pp. 1415-1425.

[3] L. H. Etzkorn, S. Gholston, W. Hughes, A semantic entropy metric. Journal of Software Maintenance and Evolution: Research and Practice, 14 (2002), pp. 1-18.

[4] R. PRESSMAN, Software engineering: a practitioner's approach (6th ed), McGraw-Hill (2005), New York.
[5] L. H. ETZKorn, H. Delugach, Towards a semantic metrics suite for object-oriented design. Proceedings of the 34th International Conference on Technology of Object-Oriented Languages and Systems (TOOLS USA 34), Institute of Electrical and Electronics Engineers (IEEE) Computer Society Press, (2000), pp. 71-80.

[6] M. SPEnKE, C. BEILKIN, An overview of GINA à the generic interactive application. Proceedings of the Workshop on User Interface Management Systems and Environments on User Interface Management and Design, (1993), pp. 273-293.

[7] J. SMART, wxWindows, Retrieved 2005 from http: //www. wxwindows.org/.

[8] R. DAVIES, Newmat C++ Matrix Library, (2006), (pp. 17), Wellington, New Zealand, http://www.robertnz.net/ol_doc.htm/, [retrieved 23 April 2006].

[9] L. H. ETZKORn, C. DAVIS, Automatically identifying reusable components in object-oriented legacy code. Institute of Electrical and Electronics Engineers (IEEE) Computer, 30 (1997), 10, pp. 66-71.

[10] L. H. EtzKorn, J. BANsiYA, C. DAVIS, Design and complexity metrics for OO classes. Journal of Object-Oriented Programming, 12 (1999), 1, pp. 35-40.

[11] L. H. EtzKoRN, A Metrics-Based Approach to the Automated Identification of Object-Oriented Reusable Software Components. Doctoral Dissertation, The University of Alabama in Huntsville, (1997).

[12] J. SowA, Conceptual Structures: Information Processing in Mind and Machine. Addison-Wesley, Reading, Massachusetts, (1984).

[13] C. SteIn, Semantic Metrics for Source Code and Design. Doctoral Dissertation, The University of Alabama in Huntsville, (2004).

[14] C. Stein, L. H. EtzKorn, G. W. COX, P. FARringTON, S. Gholston, D. Utley, J. Fortune, Proceedings of the 1st International Workshop on Software Audit and Metrics, Porto, Portugal, (2004), pp. 49-58.

[15] B. Kitchenham, S. PleEger, N. Fenton, Towards a Framework for Software Measurement Validation. IEEE Transactions on Software Engineering, 21 (1995), 12, pp. 929-944.

[16] L. BRIAND, S. MORASCA, V. BASILI, Property-based software engineering measurement. IEEE Transactions on Software Engineering, 22 (1996), 1, pp. 68-86.

[17] Kabaili, R. Keller, F. Lustman, G. SAINT-Denis, Class cohesion revisited: an empirical study on industrial systems. Fifth European Conference on Software Maintenance and Reengineering, (2001), pp. 39-47.

[18] A. Mitchell, J. Power, An empirical investigation into the dimensions of run time coupling in Java programs. Proceedings of the 3rd International Conference on Principles and Programming in Java (PPPJ'04), Las Vegas, Nevada, (2004), pp. 9-14. 
[19] W. LI, S. HENRY, Object-oriented metrics that predict maintainability. Journal of Systems and Software, 23 (1993), 2, pp. 111-122.

[20] W. HopkINS, A new view of statistics. SportScience: Dunedin, New Zealand. Retrieved 2005 from www.sportsci.org/resource/stats//.

[21] J. COHEN, Statistical power analysis for the behavioral sciences (2nd ed). Lawrence Erlbaum Publishing, Mahwah, New Jersey, (1988).

[22] W. Dillon, M. Goldstein, Multivariate analysis: methods and applications. John Wiley and Sons, New York, (1984).

Received: January, 2006 Revised: November, 2006 Accepted: December, 2006

Contact addresses:

Glenn Cox

Department of Computer Science University of Alabama in Huntsville Huntsville, Alabama, USA e-mail: gcox@cs.uah.edu

Sampson Gholston Industrial and Systems Engineering Management Department

University of Alabama in Huntsville Huntsville, Alabama, USA

Dawn R. Utley Industrial and Systems Engineering Management Department University of Alabama in Huntsville Huntsville, Alabama, USA

Letha H. Etzkorn

Department of Computer Science University of Alabama in Huntsville Huntsville, Alabama, USA

Cara Stein Gall Department of Computer Science Edinboro University of Pennsylvania Edinboro, Pennsylvania, USA

Phillip A. Farrington Industrial and Systems Engineering Management Department

University of Alabama in Huntsville Huntsville, Alabama, USA

Julie Fortune Industrial and Systems Engineering Management Departmen

University of Alabama in Huntsville Huntsville, Alabama, USA
DR. GLENN COX is an Associate Professor in the Computer Science Department of the University of Alabama in Huntsville. He holds Ph.D., Masters, and Bachelors degrees in Electrical Engineering from Auburn University. He has published more than 40 conference and journal papers in the areas of software metrics, computer networking, modeling and simulation, and integrated circuit design. He worked for 22 years in industry prior to his academic career.

DR. SAMPSON GHOLSTON is an Associate Professor at the University of Alabama in Huntsville. He has a Ph.D. degree from the University of Alabama in Huntsville in Industrial and Systems Engineering and a Masters degree from the University of Alabama in Industrial Engineering. He has also worked for the Saturn Corporation as a Supplier Quality Engineer. His research interests include quality engineering, quality management, and applied statistics.

DR. DAwn R. UTLEy, Ph.D., P.E. is an Associate Professor at the University of Alabama in Huntsville, teaches and conducts research in the area of engineering management. Dr. Utley has worked in the specific area of quality measures for 15 years. She has a Ph.D. in Industrial and Systems Engineering (Engineering Management) from UAH, an M.S. degree in Industrial Engineering (Engineering Management) from the University of Tennessee, and a B.S. in Civil Engineering from Tennessee Technological University. She worked for six years for TVA as a structural civil engineer prior to her academic career.

DR. LETHA H. ETZKORN is an Associate Professor in the Computer Science department at the University of Alabama in Huntsville. She has published more than 60 papers in refereed journals and conferences. She teaches in several areas, including software engineering, client/server systems, and networking. Her primary research interests are in software engineering (primarily software metrics and program comprehension), and mobile and intelligent agents.

DR. CARA STEIN Gall is an Assistant Professor in the Math and Computer Science Department at Edinboro University of Pennsylvania. She received her Ph.D. in Computer Science from the University of Alabama in Huntsville. She teaches Programming, Computer Architecture, and Computer Literacy. Her research interests are in software metrics.

DR. Phillip A. FARrington is an Associate Professor in the Department of Industrial and Systems Engineering and Engineering Management at the University of Alabama in Huntsville. He holds B.S. and M.S. degrees in Industrial Engineering from the University of MissouriColumbia and a Ph.D. in Industrial Engineering and Management from Oklahoma State University. His research interests include collaborative systems engineering, integrated product development, quality engineering, and rapid simulation model development.

DR. JULIE FORTUNE holds Ph.D., M.S.E, and B.S.E. degrees in Industrial and Systems Engineering from the University of Alabama in Huntsville (UAH) and a B.S. in Biochemistry from the University of Missouri-Columbia. Dr. Fortune is a research engineer at UAH?s Center for Modeling, Simulation and Analysis (CMSA) and is also an adjunct assistant professor in the UAH Industrial and Systems Engineering and Engineering Management department. Her research expertise includes system engineering, quality engineering, software metrics, and statistical analysis. 